\title{
The Quality Of Cluster Randomized Controlled Trials Of Pediatrics Should Be Improved: An Integrative Literature Review
}

\author{
Hui Lan \\ Lanzhou University \\ Jianjian Wang \\ Lanzhou University \\ Shouyuan Wu \\ Lanzhou University \\ Qiangqiang Guo \\ Lanzhou University \\ Ping Wang \\ Lanzhou University \\ Juanjuan Zhang \\ Lanzhou University \\ Siya Zhao \\ Lanzhou University \\ Xiao Liu \\ Lanzhou University \\ Xingrong Liu \\ Lanzhou University \\ Yaolong Chen ( $\square$ chevidence@lzu.edu.cn ) \\ Lanzhou University
}

\section{Research Article}

Keywords: Pediatrics, cluster Randomized Controlled Trials, Risk of Bias

Posted Date: January 12th, 2022

DOI: https://doi.org/10.21203/rs.3.rs-1112468/v1

License: (c) (i) This work is licensed under a Creative Commons Attribution 4.0 International License. Read Full License 


\section{Abstract}

Background: The number of cluster Randomized Controlled Trials (CRCTs) have been increasing and application in pediatrics is also growing. However, we did not know the quality of CRCTs in pediatrics and no previous study has comprehensively analyzed cRCTs in pediatrics. To investigate the status of CRCTs in pediatrics and analyzed influencing factors of the methodological quality.

Methods: Through a search of 10 highest-impact-factor journals from PEDIATRICS and MEDICINE, GENERAL \& INTERNAL, we identified cRCTs published from 1 January 2015 to 7 June 2020. The associations between study factors and grade of ROB (Risk of Bias) quality were analyzed by Pearson's chi-squared test.

Results: A total of 73 cRCTs were included. For the number of cRCTs, it has fluctuated in the past five years. For the International Classification of Diseases Eleventh Revision (ICD-11), 34.2\% cRCTs could not be classified, of which more focused on vaccines; $15.1 \%$ cRCTs were classified into "X Extension Codes", of which more focused on vaccines and school bullying. For research settings, studies were concentrated in schools, communities, hospitals, clinics, and kindergartens, of which schools (36.5\%) were the most. The number of clusters ranged from 1 to 1533 (M=28) and the participants ranged from 135 to 190,238 (M=1903). For the results of ROB assessment, $31 \mathrm{cRCTs}$ were in high-quality, 28 were in low-quality, and 14 were unclear. The majority of cRCTs provided flow diagrams (87.8\%), registered (98.6\%), and made a distinction between primary and secondary outcomes (86.5\%).

Conclusions: The further improvement of the quality of CRCTs in pediatrics is needed, particularly in the terms of randomization, allocation concealment, and blinding. Writing and publishing the protocol can enhance transparency. Comply with of CONSORT statement for future studies is needed.

\section{Background}

Because of the strong argument strength, randomized trials usually help researchers determine the efficacy of interventions in evidence-based medicine ${ }^{[1]}$. The multi-center design is a common method used in clinical trials, particularly in randomized controlled trials (RCTs). It is also widely used in evaluating the intervention's efficacy because of interventions in clusters ${ }^{[2]}$, which makes it possible not only to avoid contamination ${ }^{[3]}$, but also to combine the advantages and characteristics of RCTs. Based on the above, and take into account the particular characteristics of children, CRCTs are also particularly appropriate for applications in pediatric studies. In recently, the number of cRCTs have been increasing and application in pediatrics is also growing. A crosssectional survey in 2021, however, find that both the methodological quality and reporting quality of cRCTs need to be improved, particularly the selection bias of methodological quality ${ }^{[4]}$. Currently, no previous study has comprehensively analyzed cRCTs in pediatrics; indeed, no study has identified what characteristics affecting the quality of cRCTs. Therefore, in this survey, cRCTs in the high-quality journals in the field of PEDIATRICS and MEDICINE, GENERAL \& INTERNAL were included to summarize the characteristics, to assess of quality based on the Cochrane risk of bias assessment tool (ROB), and to analyze the influencing factors of the methodological quality of cRCTs in pediatrics.

\section{Methods}

\section{Data Sources and Search}

Ten journals, namely Journal of the American Medical Association (JAMA), British Medical Journal (BMJ), Lancet, New England Journal of Medicine (NEJM), Annals of Internal Medicine, JAMA Pediatrics, Lancet Child \& Adolescent Health, Journal of the America Academy of Child and Adolescent Psychiatry, Archives of Disease in Childhood-Fetal and Neonatal and Pediatrics were systematically searched from 1 January 2015 to 7 June 2020 in PubMed. We selected the top ten journals According to the impact factors in the PEDIATRICS and MEDICINE, GENERAL \& INTERNAL in JCR 2019 (Journal Citation Reports) respectively. The search terms were "pediatric*", "paediatric*", "child*", "infant*", "adolescent*", "baby", "babies", "trottie*", "youth*”, "kids", "toddler*", "pre-

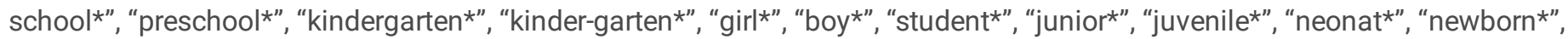
"teenager*", "pubescent", "preterm", "puberty", "young*”; "cluster", "crossover trial", "cluster-crossover trial", "cluster crossover trial", "cluster-randomized", "cluster randomized", "cluster-randomised", "cluster randomised", "cluster RCT".

\section{Selection Criteria}

Page 2/16 
Studies fulfilling all the following criteria were considered eligible: (1) the study was a CRCT; (2) the study subject was about newborn, infant, toddler, child, and adolescent; and (3) studies published in Chinese or English.

The following types of studies were excluded: (1) conference proceedings, abstracts, letters, or protocols; (2) studies for which the full text was not available; (3) studies were duplicated; and (4) RCTs at the individual level.

\section{Study Selection}

After eliminating duplicates, four researchers independently in pairs in two steps as described below. Before the formal selection, a training exercise of a random sample of 200 citations was conducted to ensure the reliability and feasibility of selection, until adequate agreement on the selecting methods was reached. Firstly, the titles and abstracts of the records were independently screened by four researchers based on selection criteria, and then the full texts of potential suitable articles were retrieved for further assessment. Disagreements were resolved by discussion or consultation with a third researcher.

\section{Data Extraction}

Using a pre-developed data extraction form, six researchers independently in pairs extracted study characteristics including: (1) Basic information: publication year, title, journal of publication, country of the first author, funding, conflicts of interest, study topic, ICD-11 classification, the country in which the study was conducted, study setting, and whether multi-center; (2) Study design: whether the trial registered, wrote a protocol, published protocol, had a diagram, conducted repeat observations, conducted evaluator training, conducted additional analyses, distinguished primary and secondary outcome indicators (yes or no), and type of intervention, number of centers, number of participants.

\section{Quality of Evidence Assessment}

Six researchers independently evaluated the methodological quality of included studies using the Cochrane risk of bias assessment tool ${ }^{[5]}$ in pairs. Disagreements are resolved by consensus or consultation with a third researcher. The overall Cochrane ROB tool grades methodological quality of included studies as high, low, and unclear ${ }^{[6,7]}$ according to the risk of bias of following 8 items: sequence generation (randomization), allocation concealment, blinding of participant, blinding of personnel, blinding of outcome assessors, incomplete outcome data, selective outcome reporting and other sources of bias. See Table 1 The grade of ROB for details.

Table 1 The grade of ROB

\section{The grade of methodological Conditions quality}

High Quality Two or more of the five key items ${ }^{a}$ have low bias, and three common items ${ }^{b}$ without high bias.

Low Quality

One or less of the five key items with low bias, and regardless of other common items.

Unclear Other conditions.

a: The five key items included sequence generation, allocation concealment, blinding of participant , blinding of personnel , blinding of outcome assessors.

b: three common items were incomplete outcome data, selective outcome reporting, and other sources of bias.

\section{Statistical Analysis}

We established the database with Microsoft Excel 2019 software, and descriptive statistics were performed by SPSS software, version 25.0 (Statistical Package for the Social Sciences version 25.0). Qualitative variables were described by absolute numbers (percentages). Quantitative variables were expressed as mean \pm standard deviation (SD). We then conducted chi-square test to assess the associations between study factors and rates of ROB. A difference was considered statistically significant effect when the $P$-value equal to or less than 0.05 . 


\section{Financial Support}

The funding source had no role in design of study, the collection, analysis, interpretation, and the decision to approve publication of the finished manuscript.

\section{Ethical Approval}

Not applicable. All the work was developed using published data.

\section{Results}

\section{Study Selection}

We identified 5,147 records from database. A total of 73 cRCTs were included eventually. More details of the search and selection are shown in Figure 1 Flow chart summarizing the process of identifying the eligible studies.

\section{Study Characteristics}

The number of included cRCTs fluctuated from 2015 to 2020 . The 73 cRCTs included 6,391 clusters ( $\mu=89, S D=213$ ) and 863,916 participants $(\mu=11,834, S D=32,339)$. Most cRCTs were from Pediatrics $(27,37.0 \%)$, Lancet $(12,16.4 \%)$, and JAMA Pediatrics (13, $17.8 \%)$. Most cRCTs were focused on vaccines $(11,15.1 \%)$ and growth and obesity of child and adolescent $(11,15.1 \%)$. According to ICD-11, most cRCTs cannot be classified $(25,34.2 \%)$, of which more focused on vaccines $(5,20 \%)$; many cRCTs focused on the most was "X Extension Codes" (11, 15.1\%), of which more focus on vaccines $(6,54.5 \%)$; then a part of studies concerned about "Endocrine, nutritional or metabolic diseases" (9, 12.3\%) and "Certain infectious or parasitic diseases" (7, 9.6\%). Most studies were from United States $(31,42.5 \%)$ and United Kingdom (56.8\%), and there were only 3 cRCTs from China (4.1\%). The first author was mostly from the United States $(35,48.0 \%)$ and the United Kingdom $(12,16.4 \%)$. Studies were conducted in community $(25,34.2 \%)$ and school $(25,34.2 \%)$, hospital $(17,23.3 \%)$, clinic $(5,6.8 \%)$, and nursery $(1,1.4 \%)$. All 73 cRCTs had financial support. More details of characteristics are shown in Table 2 Characteristics of included studies at the end of the text.

Table 2 Characteristics of included studies 


\begin{tabular}{|c|c|c|c|c|c|c|c|}
\hline Study ID & $\begin{array}{l}\text { First } \\
\text { author's } \\
\text { country }\end{array}$ & Research topic & ICD-11 & Country & Setting & $\begin{array}{l}\text { Cluster } \\
\text { (n) }\end{array}$ & $\begin{array}{l}\text { Participants } \\
\text { (n) }\end{array}$ \\
\hline $\begin{array}{l}\text { Weisleder } \\
2015\end{array}$ & America & $\begin{array}{l}\text { Abusive } \\
\text { relationship of } \\
\text { adolescent }\end{array}$ & $\begin{array}{l}23 \text { External causes } \\
\text { of morbidity or } \\
\text { mortality }\end{array}$ & America & School & 7 & 1,012 \\
\hline $\begin{array}{l}\text { Yousafza } \\
2015\end{array}$ & Pakistan & Parenting skills & $\begin{array}{l}24 \text { Factors } \\
\text { influencing health } \\
\text { status or contact } \\
\text { with health services }\end{array}$ & Pakistan & Community & 1 & 1,489 \\
\hline $\begin{array}{l}\text { Sikander } \\
2015\end{array}$ & America & $\begin{array}{l}\text { Vaccination } \\
\text { reminder }\end{array}$ & Unable to classify & America & Clinic & 4 & 6,593 \\
\hline Kempe 2015 & China & Sleep & Unable to classify & $\begin{array}{l}\text { Hong Kong, } \\
\text { China }\end{array}$ & School & 15 & 8,856 \\
\hline Pinder 2015 & Pakistan & Breast feeding & Unable to classify & Pakistan & Community & 40 & 454 \\
\hline $\begin{array}{l}\text { Andersson } \\
2015\end{array}$ & America & $\begin{array}{l}\text { Immunization } \\
\text { coverage rate }\end{array}$ & Unable to classify & America & Community & 16 & 50,549 \\
\hline Chang 2015 & America & $\begin{array}{l}\text { Children's } \\
\text { obesity }\end{array}$ & $\begin{array}{l}21 \text { Symptoms, signs } \\
\text { or clinical findings, } \\
\text { not elsewhere } \\
\text { classified }\end{array}$ & America & Clinic & 14 & 549 \\
\hline $\begin{array}{l}\text { Brotman } \\
2015\end{array}$ & China & Myopia & $\begin{array}{l}09 \text { Diseases of the } \\
\text { visual system }\end{array}$ & China & School & 12 & 1,903 \\
\hline Döring 2015 & Britain & Malaria & $\begin{array}{l}01 \text { Certain } \\
\text { infectious or } \\
\text { parasitic diseases }\end{array}$ & Gambia & Community & 70 & 7,845 \\
\hline Kempe 2015 & Mexico & $\begin{array}{l}\text { Prevention of } \\
\text { dengue fever }\end{array}$ & $\begin{array}{l}01 \text { Certain } \\
\text { infectious or } \\
\text { parasitic diseases }\end{array}$ & $\begin{array}{l}\text { Nicaragua } \rrbracket \\
\text { Mexico }\end{array}$ & Community & 150 & 20,455 \\
\hline $\begin{array}{l}\text { Mortimer } \\
2015\end{array}$ & America & $\begin{array}{l}\text { Brief } \\
\text { intervention } \\
\text { and referral }\end{array}$ & Unable to classify & America & Hospital & 52 & 5,183 \\
\hline Soofi 2015 & Jamaica & Vaccination & Unable to classify & $\begin{array}{l}\text { Jamaica, } \\
\text { Antigua, } \\
\text { Saint Lucia }\end{array}$ & Community & 29 & 707 \\
\hline Keenan 2015 & America & Vaccination & Unable to classify & America & Hospital & 56 & 347 \\
\hline Tofail 2015 & America & $\begin{array}{l}\text { Effect of } \\
\text { sedatives on } \\
\text { clinical } \\
\text { outcome in } \\
\text { children }\end{array}$ & X Extension Codes & America & Hospital & 31 & 2,449 \\
\hline $\begin{array}{l}\text { Azor- } \\
\text { Martinez } \\
2015\end{array}$ & Britain & $\begin{array}{l}\text { Reduce salt } \\
\text { intake for } \\
\text { children and } \\
\text { families } \\
\text { through } \\
\text { education }\end{array}$ & Unable to classify & China & School & 28 & 832 \\
\hline Stewart 2015 & Swedish & $\begin{array}{l}\text { Prevention of } \\
\text { Adolescent } \\
\text { suicide } \\
\text { through } \\
\text { Education }\end{array}$ & $\begin{array}{l}21 \text { Symptoms, signs } \\
\text { or clinical findings, } \\
\text { not elsewhere } \\
\text { classified }\end{array}$ & $\begin{array}{l}10 \\
\text { countries in } \\
\text { the } \\
\text { European } \\
\text { Union }\end{array}$ & School & 168 & 11,110 \\
\hline Tiono 2015 & Australia & $\begin{array}{l}\text { Cardiovascular } \\
\text { disease in } \\
\text { primary school } \\
\text { students }\end{array}$ & Unable to classify & Australia & School & 20 & 264 \\
\hline
\end{tabular}




\begin{tabular}{|c|c|c|c|c|c|c|c|}
\hline Ordway 2016 & America & $\begin{array}{l}\text { Mental health } \\
\text { and academic } \\
\text { achievement } \\
\text { of young } \\
\text { children }\end{array}$ & $\begin{array}{l}23 \text { External causes } \\
\text { of morbidity or } \\
\text { mortality }\end{array}$ & America & School & 10 & 1,050 \\
\hline Shet 2016 & Canada & $\begin{array}{l}\text { Improve pain } \\
\text { assessment } \\
\text { and } \\
\text { management } \\
\text { of hospitalized } \\
\text { children }\end{array}$ & $\begin{array}{l}21 \text { Symptoms, signs } \\
\text { or clinical findings, } \\
\text { not elsewhere } \\
\text { classified }\end{array}$ & Canada & Hospital & 16 & 1,440 \\
\hline Pullan 2016 & America & $\begin{array}{l}\text { Attention } \\
\text { deficit / } \\
\text { hyperactivity } \\
\text { disorder }\end{array}$ & $\begin{array}{l}06 \text { Mental, } \\
\text { behavioural or } \\
\text { neurodevelopmental } \\
\text { disorders }\end{array}$ & America & School & 23 & 135 \\
\hline Foy 2016 & America & Infant's weight & $\begin{array}{l}21 \text { Symptoms, signs } \\
\text { or clinical findings, } \\
\text { not elsewhere } \\
\text { classified }\end{array}$ & America & Hospital & 4 & 1,173 \\
\hline $\begin{array}{l}\text { Prendergast } \\
2016\end{array}$ & Australia & $\begin{array}{l}\text { Safety of Baby } \\
\text { Simulator in } \\
\text { preventing } \\
\text { Adolescent } \\
\text { pregnant } \\
\text { Children from } \\
\text { being } \\
\text { vaccinated }\end{array}$ & Unable to classify & Australia & School & 57 & 5,327 \\
\hline Ojha 2016 & Canada & $\begin{array}{l}\text { Safety of Baby } \\
\text { Simulator in } \\
\text { preventing } \\
\text { Adolescent } \\
\text { pregnant } \\
\text { Children from } \\
\text { being } \\
\text { vaccinated }\end{array}$ & X Extension Codes & Canada & Community & 48 & 3,451 \\
\hline $\begin{array}{l}\text { Skjerven } \\
2016\end{array}$ & Swedish & $\begin{array}{l}\text { Children's } \\
\text { obesity }\end{array}$ & $\begin{array}{l}\text { 05Endocrine, } \\
\text { nutritional or } \\
\text { metabolic diseases }\end{array}$ & Swedish & Community & 59 & 1,369 \\
\hline $\begin{array}{l}\text { Staedke } \\
2016\end{array}$ & America & Vaccine recall & X Extension Codes & America & Community & 7 & 1,422 \\
\hline Brayn 2016 & Netherland & $\begin{array}{l}\text { The influence } \\
\text { of physical } \\
\text { exercise on } \\
\text { Children's } \\
\text { academic } \\
\text { achievement }\end{array}$ & Unable to classify & Netherland & School & 12 & 499 \\
\hline Miller 2016 & America & $\begin{array}{l}\text { Physical } \\
\text { exercise }\end{array}$ & Unable to classify & America & School & 20 & 1,143 \\
\hline Wing 2016 & America & $\begin{array}{l}\text { Influenza } \\
\text { vaccine }\end{array}$ & X Extension Codes & America & School & 44 & 19,776 \\
\hline He 2016 & America & Vaccine supply & X Extension Codes & America & Hospital & 35 & 3,147 \\
\hline He 2017 & Uganda & $\begin{array}{l}\text { evaluate an } \\
\text { intervention } \\
\text { designed to } \\
\text { teach primary } \\
\text { school children } \\
\text { to assess } \\
\text { claims about } \\
\text { the effects of } \\
\text { treatments }\end{array}$ & Unable to classify & Uganda & School & 120 & 12,798 \\
\hline Wasserman & Malawi & Children's & 12 Diseases of the & Malawi & Community & 150 & 10,750 \\
\hline
\end{tabular}




\begin{tabular}{|c|c|c|c|c|c|c|c|}
\hline \multirow{2}{*}{$\begin{array}{l}2016 \\
\text { Robinson } \\
2017\end{array}$} & \multirow[b]{2}{*}{ French } & \multirow{2}{*}{$\begin{array}{l}\text { pneumonia } \\
\text { omphalitis }\end{array}$} & \multicolumn{5}{|l|}{ respiratory system } \\
\hline & & & $\begin{array}{l}19 \text { Certain } \\
\text { conditions } \\
\text { originating in the } \\
\text { perinatal period }\end{array}$ & French & Hospital & 6 & 8,698 \\
\hline $\begin{array}{l}\text { Brotman } \\
2017\end{array}$ & $\begin{array}{l}\text { New } \\
\text { Zealand }\end{array}$ & $\begin{array}{l}\text { Campus } \\
\text { bullying }\end{array}$ & X Extension Codes & $\begin{array}{l}\text { New } \\
\text { Zealand }\end{array}$ & School & 16 & 1,663 \\
\hline Pfiffner 2017 & America & $\begin{array}{l}\text { Children's } \\
\text { obesity }\end{array}$ & $\begin{array}{l}05 \text { Endocrine, } \\
\text { nutritional or } \\
\text { metabolic diseases }\end{array}$ & America & Hospital & 9 & 378 \\
\hline $\begin{array}{l}\text { Brinkman } \\
2017\end{array}$ & America & $\begin{array}{l}\text { Type } 2 \\
\text { diabetes in } \\
\text { children }\end{array}$ & $\begin{array}{l}05 \text { Endocrine, } \\
\text { nutritional or } \\
\text { metabolic diseases }\end{array}$ & America & Hospital & 4 & 1,369 \\
\hline $\begin{array}{l}\text { Mullender- } \\
\text { Wijnsma } \\
2017\end{array}$ & Pakistan & Take vitamin A & X Extension Codes & Pakistan & Community & 390 & 13,225 \\
\hline $\begin{array}{l}\text { Cradock } \\
2017\end{array}$ & America & $\begin{array}{l}\text { Vision } \\
\text { screening }\end{array}$ & $\begin{array}{l}24 \text { Factors } \\
\text { influencing health } \\
\text { status or contact } \\
\text { with health services }\end{array}$ & America & Hospital & 12 & 1,166 \\
\hline $\begin{array}{l}\text { Szilagyi } \\
2017\end{array}$ & America & Obesity & $\begin{array}{l}05 \text { Endocrine, } \\
\text { nutritional or } \\
\text { metabolic diseases }\end{array}$ & America & School & 6 & 697 \\
\hline Nsangi 2017 & America & $\begin{array}{l}\text { Infant sleep } \\
\text { habits }\end{array}$ & Unable to classify & America & Hospital & 16 & 3,733 \\
\hline Farme 2017 & Britain & $\begin{array}{l}\text { Breastfeeding } \\
\text { for asthma, } \\
\text { lung function } \\
\text { and atopic } \\
\text { eczema }\end{array}$ & $\begin{array}{l}12 \text { Diseases of the } \\
\text { respiratory system, } \\
14 \text { Diseases of the } \\
\text { skin }\end{array}$ & Belarus & Hospital & 34 & 17,046 \\
\hline Lumeng2018 & Britain & $\begin{array}{l}\text { Financial } \\
\text { incentives for } \\
\text { breastfeeding }\end{array}$ & $\begin{array}{l}19 \text { Certain } \\
\text { conditions } \\
\text { originating in the } \\
\text { perinatal period }\end{array}$ & Britain & Hospital & 92 & 10,010 \\
\hline Lloyd 2018 & Britain & $\begin{array}{l}\text { Healthy } \\
\text { lifestyle } \\
\text { prevents } \\
\text { obesity in } \\
\text { primary school } \\
\text { children }\end{array}$ & $\begin{array}{l}05 \text { Endocrine, } \\
\text { nutritional or } \\
\text { metabolic diseases }\end{array}$ & Britain & School & 32 & 1,324 \\
\hline Adab 2018 & America & vaccination & X Extension Codes & America & Hospital & 5 & 1,621 \\
\hline Bonell 2018 & America & $\begin{array}{l}\text { Azithromycin } \\
\text { reduces } \\
\text { mortality }\end{array}$ & X Extension Codes & $\begin{array}{l}\text { Malawi, } \\
\text { Niger, } \\
\text { Tanzania, }\end{array}$ & Community & 1533 & 190,238 \\
\hline Shinde 2018 & Canada & $\begin{array}{l}\text { Pediatric early } \\
\text { warning } \\
\text { system }\end{array}$ & Unable to classify & $\begin{array}{l}\text { Belgium, } \\
\text { Canada, } \\
\text { Britain, } \\
\text { Ireland, } \\
\text { Italy, New } \\
\text { Zealand, } \\
\text { Netherland }\end{array}$ & Hospital & 21 & 144,539 \\
\hline Pfiffner 2018 & America & $\begin{array}{l}\text { Effects of } \\
\text { alcohol and } \\
\text { cannabis on } \\
\text { sexually } \\
\text { transmitted } \\
\text { infections in } \\
\text { adolescents }\end{array}$ & $\begin{array}{l}01 \text { Certain } \\
\text { infectious or } \\
\text { parasitic diseases }\end{array}$ & America & $\begin{array}{l}\text { Community } \\
\text { (Detention } \\
\text { facility) }\end{array}$ & 145 & 460 \\
\hline
\end{tabular}




\begin{tabular}{|c|c|c|c|c|c|c|c|}
\hline $\begin{array}{l}\text { Polonsky } \\
2018\end{array}$ & Bangladesh & $\begin{array}{l}\text { Child } \\
\text { development }\end{array}$ & Unable to classify & Bangladesh & Community & 720 & 5,551 \\
\hline Gerald 2018 & Britain & $\begin{array}{l}\text { Childhood } \\
\text { Obesity } \\
\text { Prevention } \\
\text { Program }\end{array}$ & $\begin{array}{l}05 \text { Endocrine, } \\
\text { nutritional or } \\
\text { metabolic diseases }\end{array}$ & Britain & School & 54 & 2,462 \\
\hline $\begin{array}{l}\text { Marshall } \\
2018\end{array}$ & Spain & $\begin{array}{l}\text { Respiratory } \\
\text { infection }\end{array}$ & $\begin{array}{l}12 \text { Diseases of the } \\
\text { respiratory system }\end{array}$ & Spain & Community & 25 & 1,176 \\
\hline Link 2018 & Britain & $\begin{array}{l}\text { Campus } \\
\text { bullying }\end{array}$ & X Extension Codes & Britain, & School & 40 & 6,667 \\
\hline Miller 2018 & America & $\begin{array}{l}\text { Child } \\
\text { development }\end{array}$ & Unable to classify & Kenya & Community & 702 & 7,960 \\
\hline Sterling 2018 & $\begin{array}{l}\text { Burkina } \\
\text { Faso }\end{array}$ & Malaria & $\begin{array}{l}01 \text { Certain } \\
\text { infectious or } \\
\text { parasitic diseases }\end{array}$ & $\begin{array}{l}\text { Burkina } \\
\text { Faso }\end{array}$ & Community & 40 & 1,980 \\
\hline $\begin{array}{l}\text { Henrikson } \\
2018\end{array}$ & America & $\begin{array}{l}\text { Parenting } \\
\text { programs and } \\
\text { children's } \\
\text { obesity }\end{array}$ & $\begin{array}{l}05 \text { Endocrine, } \\
\text { nutritional or } \\
\text { metabolic diseases }\end{array}$ & America & Community & 6 & 237 \\
\hline Curley 2018 & Britain & $\begin{array}{l}\text { School } \\
\text { environment } \\
\text { affects } \\
\text { students' } \\
\text { health and } \\
\text { scholarship }\end{array}$ & Unable to classify & India & School & 75 & 13,035 \\
\hline $\begin{array}{l}\text { Stevens } \\
2018\end{array}$ & America & $\begin{array}{l}\text { Reading aloud } \\
\text { promotes } \\
\text { parent-child } \\
\text { interaction and } \\
\text { children's } \\
\text { development }\end{array}$ & Unable to classify & Brazil & $\begin{array}{l}\text { Educational } \\
\text { nursery }\end{array}$ & 12 & 612 \\
\hline Wood 2018 & America & $\begin{array}{l}\text { Collaborative } \\
\text { Lifeskills } \\
\text { Program in the } \\
\text { treatment of } \\
\text { Children with } \\
\text { attention } \\
\text { Disorder }\end{array}$ & $\begin{array}{l}06 \text { Mental, } \\
\text { behavioural or } \\
\text { neurodevelopmental } \\
\text { disorders }\end{array}$ & America & School & 23 & 135 \\
\hline Fu 2019 & America & $\begin{array}{l}\text { Mental health } \\
\text { referral }\end{array}$ & Unable to classify & America & Clinic & 6 & 342 \\
\hline Guen 2019 & America & $\begin{array}{l}\text { Breakfast } \\
\text { affects } \\
\text { children's } \\
\text { obesity }\end{array}$ & $\begin{array}{l}05 \text { Endocrine, } \\
\text { nutritional or } \\
\text { metabolic diseases }\end{array}$ & America & School & 16 & 1,362 \\
\hline Sharifi 2019 & India & $\begin{array}{l}\text { The effect of } \\
\text { parent } \\
\text { consultation } \\
\text { Education on } \\
\text { the treatment } \\
\text { rate of } \\
\text { Children's } \\
\text { anemia }\end{array}$ & $\begin{array}{l}03 \text { Diseases of the } \\
\text { blood or blood- } \\
\text { forming organs }\end{array}$ & India & Community & 56 & 1,625 \\
\hline $\begin{array}{l}\text { Hannon } \\
2019\end{array}$ & Britain & Worm infection & $\begin{array}{l}01 \text { Certain } \\
\text { infectious or } \\
\text { parasitic diseases }\end{array}$ & Kenya & Community & 120 & 147,463 \\
\hline Modest 2019 & America & Malaria & $\begin{array}{l}01 \text { Certain } \\
\text { infectious or } \\
\text { parasitic diseases }\end{array}$ & $\begin{array}{l}\text { Burkina } \\
\text { Faso }\end{array}$ & Community & 8 & 590 \\
\hline Moon 2019 & Zimbabwe & HIV infection & 24 Factors & Zimbabwe & Community & 211 & 5,270 \\
\hline
\end{tabular}




\begin{tabular}{|c|c|c|c|c|c|c|c|}
\hline & & $\begin{array}{l}\text { and anemia in } \\
\text { children }\end{array}$ & $\begin{array}{l}\text { influencing health } \\
\text { status or contact } \\
\text { with health services }\end{array}$ & & & & \\
\hline Flohr 2019 & America & Smoking & Unable to classify & America & Clinic & 18 & 8,184 \\
\hline Relton 2019 & America & Infant sleep & Unable to classify & America & Hospital & 16 & 1,263 \\
\hline Dixon 2019 & America & $\begin{array}{l}\text { Vaccination for } \\
\text { children }\end{array}$ & Unable to classify & America & Clinic & 24 & 156 \\
\hline $\begin{array}{l}\text { Parshuram } \\
2019\end{array}$ & America & $\begin{array}{l}\text { Children's } \\
\text { asthma }\end{array}$ & $\begin{array}{l}12 \text { Diseases of the } \\
\text { respiratory system, } \\
14 \text { Diseases of the } \\
\text { skin }\end{array}$ & America & School & 20 & 8,916 \\
\hline $\begin{array}{l}\text { Lumeng } \\
2020\end{array}$ & Britain & $\begin{array}{l}\text { Financial } \\
\text { incentives for } \\
\text { breastfeeding }\end{array}$ & $\begin{array}{l}19 \text { Certain } \\
\text { conditions } \\
\text { originating in the } \\
\text { perinatal period }\end{array}$ & Britain & Hospital & 92 & 10,010 \\
\hline Anokye 2020 & Australia & $\begin{array}{l}\text { Meningococcal } \\
\text { infection rate } \\
\text { of students } \\
\text { after } \\
\text { vaccination }\end{array}$ & X Extension Codes & Australia & School & 237 & 24,269 \\
\hline $\begin{array}{l}\text { Stockwell } \\
2020\end{array}$ & Britain & Child nutrition & $\begin{array}{l}05 \text { Endocrine, } \\
\text { nutritional or } \\
\text { metabolic diseases }\end{array}$ & India & Community & 56 & 2,469 \\
\hline $\begin{array}{l}\text { Taveras } \\
2020\end{array}$ & Norwegian & $\begin{array}{l}\text { Infantile } \\
\text { specific } \\
\text { dermatitis }\end{array}$ & $\begin{array}{l}14 \text { Diseases of the } \\
\text { skin }\end{array}$ & $\begin{array}{l}\text { Norwegian, } \\
\text { Swedish }\end{array}$ & Community & 92 & 2,397 \\
\hline Coker 2020 & America & Mental illness & Unable to classify & America & School & 14 & 751 \\
\hline $\begin{array}{l}\text { Nabi-Burza } \\
2020\end{array}$ & America & $\begin{array}{l}\text { Prevention of } \\
\text { adolescent } \\
\text { abuse }\end{array}$ & Unable to classify & America & School & 48 & 973 \\
\hline Opel 2020 & Britain & Malaria & $\begin{array}{l}01 \text { Certain } \\
\text { infectious or } \\
\text { parasitic diseases }\end{array}$ & Uganda & Community & 104 & 27,817 \\
\hline
\end{tabular}

Note: ICD-11 is International Classification of Diseases 11th Revision.

\section{Quality of Evidence Assessment}

There were variabilities across various RoB items. A total of 31 (42.5\%) had high quality, 28 (38.4\%) had low quality, 14 (19.2\%) had unclear. Across five key RoB items, including randomization, allocation concealment, blinding of participant, blinding of personnel and blinding of outcome assessors, of which the blinding of personnel and blinding of participant had the highest bias. From the randomization process, there were $50(68.5 \%)$ trials have high bias, and others have unclear. From the allocation concealment, there were $39(53.4 \%)$ studies have unclear, $28(38.4 \%)$ have low bias and only $6(8.2 \%)$ have high bias. From the blinding of participant and personnel, there were 34 (46.6\%) studies have unclear, 26 (35.6\%) have high bias, and 13 (17.8\%) have low bias. From the blinding of outcome assessors, there were 38 (52.1\%) studies have low bias, 26 (35.6\%) have unclear, and 9 (12.3\%) have high bias. Across three common RoB items, including incomplete data, reporting bias, and other bias, of which the other bias had the lowest bias. From the incomplete outcome data, there were 59 (80.82\%) have low bias, 9 (12.33\%) have high bias, and 5 (6.85\%) have unclear. From the selective outcome reporting, there were $63(86.30 \%)$ have low bias, and others $(13.70 \%)$ were high bias. From the other sources of bias, there were $65(89.04 \%)$ have low bias, 7 (9.59\%) have high bias, and only $1(1.37 \%)$ has unclear. More details are shown in Table 3 The results of ROB assessment.

Table 3 The results of ROB assessment 


\begin{tabular}{|llll|}
\hline Items & Low bias [n (\%)] & Unclear [n (\%)] & High bias [n (\%)] \\
\hline Randomization & $23(31.51)$ & $50(68.49)$ & $0(0.00)$ \\
\hline Allocation concealment & $28(38.36)$ & $39(53.42)$ & $6(8.22)$ \\
\hline Blinding of participant & $9(12.33)$ & $41(56.16)$ & $23(31.51)$ \\
\hline Blinding of personnel & $13(17.81)$ & $37(50.68)$ & $23(31.51)$ \\
\hline Blinding of outcome assessors & $38(52.05)$ & $26(35.62)$ & $9(12.33)$ \\
\hline Incomplete outcome data & $59(80.82)$ & $5(6.85)$ & $9(12.33)$ \\
\hline Selective outcome reporting & $63(86.30)$ & $0(0.00)$ & $10(13.70)$ \\
\hline Other sources of bias & $65(89.04)$ & $1(1.37)$ & $7(9.59)$ \\
\hline
\end{tabular}

\section{Comparisons of ROB Between Groups}

We evaluated the association between each of the trial characteristics on trial quality. The Chi-square test showed that produced a protocol has a statistically significant impact on the methodological quality of $\mathrm{CRCTs}(O R=0.261, P=0.014)$, and other trial factors had no statistically significance. See Table 4 Chi-square analysis results of each research factorat the end of the text for details.

Table 4 Chi-square analysis results of each research factor 


\begin{tabular}{|c|c|c|c|}
\hline Research factors & OR & $95 \% \mathrm{Cl}$ & $P$-value \\
\hline Protocol produced & & & 0.014 \\
\hline Yes & 1.000 & & \\
\hline No & 0.261 & $0.087-0.783$ & \\
\hline Repeat observations conducted & & & 0.562 \\
\hline Yes & 1.000 & & \\
\hline No & 0.733 & $0.257-2.095$ & \\
\hline Additional analysis conducted & & & 0.141 \\
\hline Yes & 1.000 & & \\
\hline No & 0.458 & $0.160-1.305$ & \\
\hline Primary and secondary outcomes distinguished & & & 0.071 \\
\hline Yes & 1.000 & & \\
\hline No & 0.207 & $0.039-1.098$ & \\
\hline \multicolumn{4}{|l|}{ Single vs. multicenter } \\
\hline Single-center & 1.000 & & \\
\hline Multicenter & 0.686 & $0.226-2.070$ & 0.505 \\
\hline Not reported & 1.800 & $0.139-23.374$ & 0.571 \\
\hline \multicolumn{4}{|l|}{ Staff training conducted } \\
\hline Yes & 1.000 & & \\
\hline No & 0.348 & $0.034-3.584$ & 0.612 \\
\hline Not reported & 0.959 & $0.214-4.292$ & 0.626 \\
\hline \multicolumn{4}{|l|}{ Protocol published } \\
\hline Yes & 1.000 & & \\
\hline Not reported & 3.684 & $0.678-20.012$ & 0.151 \\
\hline Not applicable & 4.200 & $0.586-30.095$ & 0.197 \\
\hline Flow diagram conducted & & & 0.148 \\
\hline Yes & 1.000 & & \\
\hline No & l & / & \\
\hline Registration reported & & & 0.325 \\
\hline Yes & 1.000 & & \\
\hline No & / & / & \\
\hline
\end{tabular}

Note: The OR of all research factors is not calculated because the value of these research factors contains 0 can not be calculated.

\section{Discussion}

In this cross-sectional survey of cRCTs published in 10 top journals in recent years. We found that the number of included cRCTs fluctuated in past five years, the fields of concern were wide, and the methodological quality of cRCTs still needed to be improved. 
We found that the number of CRCT literatures in pediatrics in recent five years fluctuated, which may be related to the reason that we only included literatures published in Chinese and English, and exclude the literatures that inability to obtain full-text and those none periodical. The reasons listed above may affected the amount of literature in different years, thus affecting the overall trend. Another reason is that we limited retrieval time to June 2020. Whatever the publishing trend, we can find that the pediatric field has continued to pay attention to the application of CRCTs in recent years. According to the data released by WHO, by the end of 2018, the top three diseases in the mortality of children under the age of five were acquired immunodeficiency syndrome, diarrhoeal disease, and tetanus ${ }^{[8]}$. It can be seen that children's health and life safety still threatened by infectious diseases. However, vaccination is the key to reduce child mortality in low and middle-income countries ${ }^{[9-11]}$. In this study, we found that most cRCTs pay high attention to vaccines, which is consistent with the above situation. In addition, obesity has always been the focus of public health all the world ${ }^{[12,13]}$, which is also consistent with the more attention paid to the development and obesity of children and adolescents in this study.

In this study, we found that the quality of CRCTs still needs to be improved. There were few descriptions of randomization, allocation concealment, and blinding in CRCTs. The risk of bias is generally high, from previous studies we found that more than half of RCTs do not implement appropriate blinding or undescribed blinding ${ }^{[14-18]}$. A study in 2021 showed that a better methodological design is needed to improve the quality of $\mathrm{CRCTs}$, such as calculation of sample size and balance randomization [19]. Therefore, we can conclude that the methodological quality of CRCTs still needs to be improved. In addition, the reporting quality of cRCTs should be improved too. From the previous study, we found that the reporting of RCTs is still insufficient ${ }^{[20]}$ and imperfect ${ }^{[21]}$, which is difficult to provide readers with necessary information. Published RCTs rarely reported the details of blinding and allocation concealment ${ }^{[22-24]}$. The same as CRCTs, a study published in 2021 found that the current reporting quality of multicenter RCTs is not optimal when evaluating the reporting quality of cRCTs from 1975 to 2021 [25].

With regard to the influencing factors, more than half of the literatures in this study wrote a protocol. The protocol is the basis for clinical trial planning, implementation, reporting, and evaluation ${ }^{[26]}$. Furthermore, the Consolidated Standards Of Reporting Trials statement 2010 (CONSORT) extension to cluster randomized trials stated that the protocol should be written in advance, registered on the clinical trial registration, and the complete version of the protocol should be provided in the text. Moreover, strictly following the CONSORT statement can improve the reporting quality ${ }^{[27]}$ and provide readers with more comprehensive information. In this study, we found that the quality of trials with protocol was higher than that of without, which was consistent with the above researches. CRCTs are prone to loss of follow-up and missing data during the implementation of the study due to a large number of participants and long follow-up time. In this situation, some studies suggested to reduce bias and improve the quality of studies by using intention analysis and sensitivity analysis in the analysis of cRCTs ${ }^{[28]}$. This study found that most of the pediatric cRCTs reported the flowcharts and adopted the additional analyses. However, the quality difference between studies was small, because included studies were from high impact factor journals, thus we didn't find out the impact of the flowcharts or use of additional analyses on the research quality.

Based on the findings of this study, we put forward the following suggestions for carrying out pediatric cRCT researches, which is also applicable to other disciplines to a certain extent. Firstly, we suggest that cRCTs should write a protocol to help the effective implementation of the research and increase the transparency of the research. Secondly, we suggest researchers should fully master the methodology of the trial, especially the implementation of a strict randomization, allocation concealment and blinding before the implementation of cRCTs, epidemiological professionals or methodologists can also be included in the trials for full guidance. Finally, we suggested to increase the training and use of CONSORT and its extended version for researchers, and further urge the standardized reporting of the trials.

\section{Strengths and Limitations}

This study has several strengths. We performed a comprehensive analyses of CRCTs in the field of pediatrics for the first time as we know. The blinding is divided into the blinding of participant, blinding of personnel and the blinding of outcome assessors, instead of the joint evaluation method when assessing the risk of bias of the study. In this way, we increased the reliability of the evaluation results ${ }^{[29]}$. In order to provide references and suggestions for future research, we also analyzed the factors affecting the ROB of cRCTs. At the same time, this study has several limitations. Firstly, the evaluation of the current research hotspots in the field of pediatrics may be affected, that many included studies could not be distinguished to the specific disease classification according to

Page $12 / 16$ 
ICD-11, of which $34.2 \%$ cRCTs involved diseases that could not be classified, and $15.1 \%$ cRCTs involved diseases classified as " $X$ Extension Codes". In addition, we may omit some important factors that affect the research quality, due to the cRCTs included in this study are all from high impact factor journals and the methodological quality heterogeneity between studies is small.

Furthermore, the analysis results may be affected by publication bias, for this study excluded the literatures published in no Chinese and English.

\section{Conclusion}

Overall, nearly half of the pediatric cRCTs published in journals with high impact factors in the past five years are of high quality. However, the five key items of the ROB still need to be improved. As to increase the standardization and normalization of cRCTs and eventually improve the quality, we recommended to write a protocol before the experiment, strengthen methodological training for researchers, and in accordance with the CONSORT statement when reporting.

\section{Abbreviations}

$\mu$ : Mean

M: Median

SD: Standard Deviation

CRCT: Cluster randomized controlled trial

CONSORT: Consolidated Standards Of Reporting Trials statement

ROB: Risk of bias

RCT: Randomized controlled trial

WHO : World Health Organization

\section{Declarations}

\section{Acknowledgements}

Not applicable.

\section{Funding}

This work was supported by the Fundamental Research Funds for the Central Universities (<Grant Number. Izujbky-2021-ey13>). No authors received other funding. The funding source had no role in design of study, the collection, analysis, interpretation, and the decision to approve publication of the finished manuscript.

\section{Availability of data and material}

Data Availability Statement: The raw data of this study come from data extraction and ROB results. The extracted data is shown in Table 2, and the ROB assessment results of every single study are shown in additional file 1.

\section{Ethics approval and consent to participate}

Not applicable

\section{Consent for publication}

Not applicable. 
Competing Interests: The authors declare no conflicts of interest.

\section{Author contributions}

Hui Lan: Data extraction, quality assessment, statistical analysis, wrote the draft and the full text. Jianjian Wang: Searched and screened researches, data extraction, quality assessment, draft modification. Shouyuan Wu: Data extraction, quality assessment, draft modification. Qiangqiang Guo: Data extraction, quality assessment, draft modification. Ping Wang: Data extraction, quality assessment. Juanjuan Zhang: Data extraction, quality assessment. Siya Zhao: Screened researches. Xiao Liu: Screened researches. Xingrong Liu: Study design, methodology support, supervision, manuscript modification. Yaolong Chen: Study design, methodology support, supervision, manuscript modification, funding acquisition.

\section{References}

1. Sackett DL, Rosenberg WM, Gray J A, Haynes RB, Richardson WS. Evidence based medicine: what it is and what it isn't (editorial). BMJ. 1996; 312(7023): 71-2.

2. Van Breukelen GJ. and Candel MJ. Calculating sample sizes for cluster randomized trials: we can keep it simple and efficient! J Clin Epidemiol. 2012; 65(11): 1212-8.

3. Easter C, Thompson JA, Eldridge S, Taljaard M, Hemming K. Cluster randomized trials of individual-level interventions were at high risk of bias. J Clin Epidemiol. 2021; 138: 49-59.

4. Bolzern J, Mnyama N, Bosanquet K, Torgerson DJ. A review of cluster randomized trials found statistical evidence of selection bias. J Clin Epidemiol. 2018; 99: 106-12.

5. Higgins JP, Altman DG, Gøtzsche PC, Jüni P, Moher D, Oxman AD, et al. The Cochrane Collaboration's tool for assessing risk of bias in randomised trials. BMJ. 2011; 343: d5928.

6. Savović J, Jones HE, Altman DG, Harris RJ, Jüni P, Pildal J, et al. Influence of reported study design characteristics on intervention effect estimates from randomized, controlled trials. Ann Intern Med. 2012; 157(6): 429-38.

7. Shea BJ, Reeves BC, Wells G, Thuku M, Hamel C, Moran J, et al. AMSTAR 2: a critical appraisal tool for systematic reviews that include randomised or non-randomised studies of healthcare interventions, or both. BMJ. 2017; 358: j4008.

8. World Health Organization: Available from: https://www.who.int/data/gho/data/themes/mortality-and-global-healthestimates(2021). Accessed 15 October 2021.

9. Lee L A, Franzel L, Atwell J, Datta SD, Friberg IK, Goldie SJ, et al. The estimated mortality impact of vaccinations forecast to be administered during 2011-2020 in 73 countries supported by the GAVI Alliance. Vaccine. 2013; 31 Suppl 2: B61-72.

10. Whitney CG, Zhou F, Singleton J, Schuchat A. Benefits from immunization during the vaccines for children program era - United States, 1994-2013. MMWR Morb Mortal Wkly Rep. 2014; 63(16): 352-5.

11. Roush SW, Murphy TV. Historical comparisons of morbidity and mortality for vaccine-preventable diseases in the United States. JAMA. 2007; 298(18): 2155-63.

12. Van Wijhe M, McDonald SA, De Melker HE, Postma MJ, Wallinga J. Effect of vaccination programmes on mortality burden among children and young adults in the Netherlands during the 20th century: a historical analysis. Lancet Infect Dis. 2016; 16(5): 592-8.

13. NCD Risk Factor Collaboration (NCD-RisC). Worldwide trends in body-mass index, underweight, overweight, and obesity from 1975 to 2016: a pooled analysis of 2416 population-based measurement studies in 128.9 million children, adolescents, and adults. Lancet. 2017; 390: 2627-42.

14. Wayant C, Tritz D, Horn J, Crow M, Vassar M. Evaluation of Risks of Bias in Addiction Medicine Randomized Controlled Trials. Alcohol Alcohol. 2021; 56(3): 284-90.

15. Kim SY, Lee EJ, Jeon JH, Kim JH, Jung IC, Kim YI. Quality Assessment of Randomized Controlled Trials of Moxibustion Using STandards for Reporting Interventions in Clinical Trials of Moxibustion (STRICTOM) and Risk of Bias (ROB). J Acupunct Meridian Stud. 2017; 10(4): 261-75.

16. Al-Qadhi G, Aboushady I, Al-Sharabi N. The Gingiva from the Tissue Surrounding the Bone to the Tissue Regenerating the Bone: A Systematic Review of the Osteogenic Capacity of Gingival Mesenchymal Stem Cells in Preclinical Studies. Stem Cells Int.

Page $14 / 16$ 
2021; 2021: 6698100.

17. Dean Walton, David C Spencer, Sarah J Nevitt, Benedict D Michael. Transcranial magnetic stimulation for the treatment of epilepsy. Cochrane Database Syst Rev. 2021; 4(4):CD011025.

18. Van Driel ML, De Sutter Al, Thorning S, Christiaens T. Different antibiotic treatments for group A streptococcal pharyngitis. Cochrane Database Syst Rev. 2021; 3(3): CD004406.

19. Parker K, Nunns M, Xiao Z, Ford T, Ukoumunne OC. Characteristics and practices of school-based cluster randomised controlled trials for improving health outcomes in pupils in the United Kingdom: a methodological systematic review. BMC Med Res Methodol. 2021; 21(1): 152.

20. Turner L, Shamseer L, Altman DG, Weeks L, Peters J, Kober T. Consolidated standards of reporting trials (CONSORT) and the completeness of reporting of randomised controlled trials (RCTs) published in medical journals. Cochrane Database Syst Rev. 2012; 11(11): Mr000030.

21. Cook DJ, Rutherford WB, Scales DC, Adhikari NKJ, Cuthbertson BH. Rationale, Methodological Quality, and Reporting of ClusterRandomized Controlled Trials in Critical Care Medicine: A Systematic Review. Crit Care Med. 2021; 49(6): 977-87.

22. Moher D, Hopewell S, Schulz KF, Montori V, Gøtzsche PC, Devereaux PJ, et al. CONSORT 2010 explanation and elaboration: updated guidelines for reporting parallel group randomised trials. Int J Surg. 2012; 10(1): 28-55.

23. Polit DF. Blinding during the analysis of research data. Int J Nurs Stud. 2011; 48(5): 636-41.

24. Polit DF, Gillespie BM, Griffin R. Deliberate ignorance: a systematic review of blinding in nursing clinical trials. Nurs Res. 2011; 60(1): 9-16.

25. Zhang X, Lam WC, Liu F, Li M, Zhang L, Xiong W, et al. A Cross-sectional literature survey showed the reporting quality of multicenter randomized controlled trials should be improved. J Clin Epidemiol. 2021; 137: 250-61.

26. Chan AW, Tetzlaff JM, Altman DG, Laupacis A, Gøtzsche PC, Krle A, et al. SPIRIT 2013 Statement: defining standard protocol items for clinical trials. Rev Panam Salud Publica. 2015; 38(6): 506-14.

27. Campbell MK, Piaggio G, Elbourne DR, Altman DG. Consort 2010 statement: extension to cluster randomised trials. BMJ. 2012; 345: e5661.

28. Li P and Stuart EA. Best (but oft-forgotten) practices: missing data methods in randomized controlled nutrition trials. Am J Clin Nutr. 2019; 109(3): 504-8.

29. Barcot O, Boric M, Dosenovic S, Puljak L. Assessing the risk of performance and detection bias in Cochrane reviews as a joint domain is less accurate compared to two separate domains. BMC Med Res Methodol. 2021; 21(1): 149.

\section{Figures}




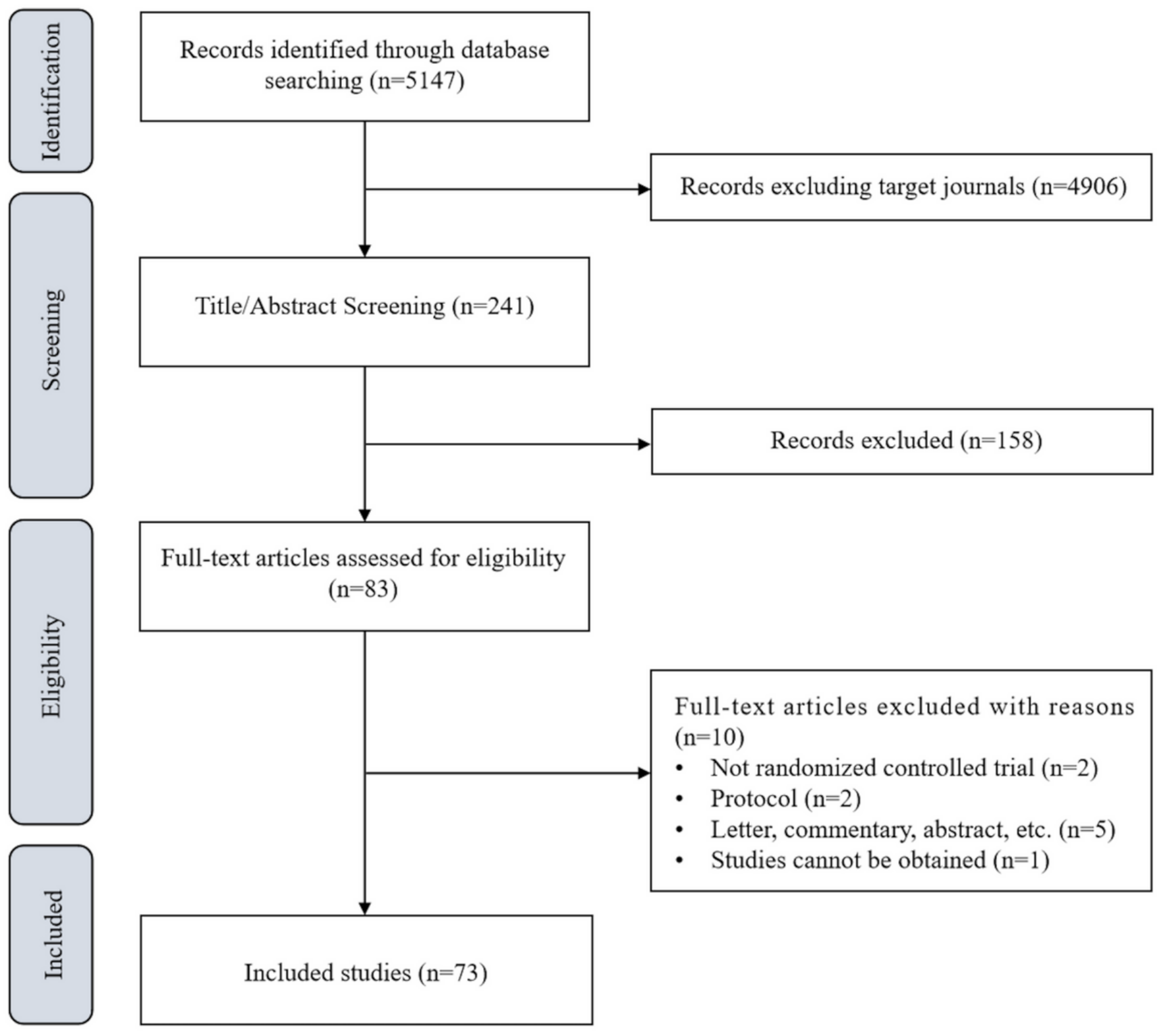

Figure 1

Flow chart summarizing the process of identifying the eligible studies.

\section{Supplementary Files}

This is a list of supplementary files associated with this preprint. Click to download.

- Additionalfile1ROBassessmentresults.xlsx 